\title{
miR-148a-3p inhibits DNMT1-mediated methylation of VEGF and promotes proliferation and invasion in glioma cells via the PI3K/Akt/eNOS signaling pathway
}

Jun-feng Huo ( $\square$ jun_fenghuo@126.com )

Huaihe Hospital of Henan University https://orcid.org/0000-0001-6460-3570

Xiao-bing Chen

Huihe Hospital of Henan University

Primary research

Keywords: Glioma; miR-148a-3p; DNMT1; VEGF; the PI3K/Akt/eNOS pathway; U87 cells

Posted Date: March 24th, 2020

DOI: https://doi.org/10.21203/rs.3.rs-18747/v1

License: (c) (1) This work is licensed under a Creative Commons Attribution 4.0 International License.

Read Full License 


\section{Abstract}

Background: Abnormal DNA methylation in the promoter region of vascular endothelial growth factor (VEGF) has been observed in multiple types of cancer. Increasing evidence shows that miR-148a-3p is involved in the regulation of methylation. However, it is unclear how miR-148a-3p regulates VEGF methylation.

Methods: Methylation-specific polymerase chain reaction (MSP) and bisulfate-sequencing PCR (BSP) were performed to detect the methylation level of the VEGF promoter region in glioma tissues and cell lines. Then, we used RT-qPCR to detect the expression of miR-148a-3p and VEGF in glioma tissues and cell lines. Human glioma U87 cells were transfected with miR-148a-3p mimic or a pcDNA3.1 overexpression vector of VEGF, and then, the proliferation, invasion and apoptosis of U87 cells were examined with cell counting kit-8 (CCK-8), Transwell assay and Flow cytometry, respectively. We next examined the relationship between DNA methyltransferase 1 (DNMT1) and miR-148a-3p with online prediction and luciferase reporter gene experiments. Furthermore, we also used Western blotting to detect the protein levels of VEGF, DNMT1, PI3K, Akt and eNOS.

Results: Both miR-148a-3p and VEGF were significantly up-regulated, and the promoter methylation of VEGF was hypomethylation in glioma. Overexpression miR-148a-3p or VEGF promoted the proliferation and invasion of U87 cells and inhibited the apoptosis. Silencing VEGF inhibited U87 cells proliferation and invasion and induced the apoptosis. Furthermore, miR-148a-3p regulated DNMT1 at the posttranscriptional. Overexpression of DNMT1 mediated an increase in the methylation level of VEGF, which reduced the expression of VEGF in U87 cells. The opposite was exact when DNMT1 was silenced. We also observed that silencing VEGF inhibited the activation of the PI3K/Akt/eNOS pathway and induced U87 cell apoptosis.

Conclusion: These results indicated that miR-148a-3p down-regulates VEGF methylation by targeting DNMT1 and promotes the proliferation, invasion of the glioma cell through the PI3K/Akt/eNOS pathway.

\section{Background}

Glioma is the most common form of a primary brain tumor in adults and one of the most invasive and lethal types of cancer in humans with a poor prognosis[1]. Despite advances in treatment strategies in recent years, including surgical resection combined with radiotherapy and chemotherapy, more than $70 \%$ of glioma patients succumb to the disease within 2 years of diagnosis, and fewer than $5 \%$ survive within 5 years $[2,3]$. The illness of glioma has a poor prognosis and high lethality, that is due primarily to the highly invasive/migratory nature of glioma cells, which can extensively infiltrate and migrate in surrounding normal tissues $[4,5]$. Therefore, to control the high lethality of glioma, it is necessary to provide a new strategy for the treatment of human glioma.

Vascular endothelial growth factor (VEGF) is a multifaceted cytokine and belongs to a large family of proteins[6]. It promotes embryonic and postnatal neovascularization[7], maintains heterogeneity of 
endothelial cell and organ[8], repairs ischemic tissues and injured organs[9]. VEGF signaling is critical for initiating tumor angiogenic responses and mediates the survival and proliferation of malignant cells[10]. It has reported that LY294002 was down-regulated VEGF expression by the PI3K/Akt pathway to prevent retinopathy of prematurity in vivo and vitro[11]. The down-regulation of VEGF is a key to the mechanisms underlying the inhibition of the development and progression of endometrial carcinoma[12]. Silencing VEGF has inhibited cell proliferation and angiogenesis in human osteosarcoma[13] and also suppressed the occurrence of lung cancer[14]. Other evidence suggests that the expression of VEGF is regulated by its methylation, hypermethylation of VEGF caused a decrease in the expression of $\operatorname{mRNA}[15,16]$. In atherosclerotic, folic acid reduced atherosclerotic lesion through elevating DNA methyltransferase activity and expression, altering MCP1 and VEGF promoter methylation, and inhibiting MCP1 and VEGF expression[17]. The expression of VEGF was significant up-regulated in human glioma via[18, 19]. Therefore, we have hypothesized that the development and progression of glioma was inhibited by elevated levels of VEGF methylation.

It is widely recognized that aberrant miRNAs are involved in various pathogenetic processes by the regulation of many signaling pathways $[12,14,20]$. In glioma, miRNAs are deeply involved in the regulation of the PI3K/AKT/eNOS signaling pathways[21, 22]. miR-148a-3p is one of the new stars of miRNAs, that with 68 nucleotide sequences is located on chromosome 7p15.2. MiR-148a performs the common functions of many miRNA species and is implicated in a series of biological processes, including cellular proliferation, apoptosis, metastasis and invasion, and is associated with the occurrence of various diseases[23-25]. In breast cancer cells, the expression of estrogen receptor- $a$ (ER- $a$ ) is an essential marker for prognosis, and miRNA-148a regulates the expression of the ER-a through DNMT1mediated DNA methylation[26]. In glioma, miR-148a to promote proliferation and metastasis of glioblastoma via targeting CADM1-miR-148a, and the occurrence of glioma is induced by overexpression of mir-148a[27]. In view of this, miR 148a-3p was identified as a potential target for the treatment of glioma. This study, we conducted to explore the underlying mechanism of regulation of glioma by miR148a-3p and VEGF methylation.

\section{Material And Methods}

\section{Tissue samples collection}

Glioma tumor tissues and adjacent non-neoplastic tissues were collected from patients who were diagnosed with glioma by a pathologist, and none of them received any anticancer therapies, such as radiotherapy, chemotherapy or molecular targeted therapy, before underwent surgery at the Huaihe Hospital of Henan University (Kaifeng, China). All samples were stored in a freezer at $-80^{\circ} \mathrm{C}$. All participants have received informed consent.

\section{Cell Culture}

The human normal glial cell lines of HEB and the human glioma U87 cell lines were purchased from American Type Culture Collection. The cells were maintained in Dulbecco's modified Eagle medium 
(DMEM) with $10 \%$ fetal bovine serum (FBS, Hyclone) and antibiotics $(100 \mu \mathrm{g} / \mathrm{ml}$ of streptomycin and 100 $\mathrm{U} / \mathrm{ml}$ of penicillin) in a humidified incubator at $37^{\circ} \mathrm{C}$ under $5 \% \mathrm{CO}_{2}$.

\section{Cell transfection}

A pcDNA3.1 overexpression vector constructed using a full-length cDNA sequence of VEGF or DNMT1 subjected to competent cell transformation. Then, the pcDNA3.1 overexpression vector was extracted using a commercial kit and identified via sequencing. MiR-148a-3p mimic and the short interfering RNA against DNMT1 (DNMT1 siRNA) or VEGF (VEGF siRNA) were designed, synthesized, and validated. The U87 cells were plated onto 24 -well plates at a density of $3 \times 10^{4}$ cells/well. When U87 cells reached $60 \%-80 \%$ confluence, the vectors or siRNAs were transfected into U87 cells by using the Lipofectamine 2000 (Invitrogen) based on the manufacturer's instruction.

\section{Dual-luciferase reporter gene assay}

MicroRNA.org (http://www.targetscan.org/) predicted the targeting relationship between miR-148a-3p and DNMT1 and was verified by the dual-luciferase reporter gene. The cells at the logarithmic growth phase were seeded into 24-well plates in triplicate. When the cell density reached $70 \%$, the cells were transfected with Lipofectamine 2000, and the DNMT1-3'UTR-wild type, DNMT1-3' UTR-mutant and miRNA-148a-3p plasmids were mixed and co-transfected into cells. After $6 \mathrm{~h}$ of culture, culture in new medium containing $10 \%$ fetal bovine serum (FBS) for $48 \mathrm{~h}$. After $48 \mathrm{~h}$ transfected, the medium was aspirated, and the DNA samples were washed twice with PBS and mixed with $100 \mu \mathrm{L}$ of passive lysis buffer (PLB) at room temperature. Fifteen minutes after, cell lysates were collected. After pre-reading for 2 seconds, $20 \mu \mathrm{L}$ of Luciferase Assay Reagent II Stop \& Glo® was added to $100 \mu \mathrm{L}$ of each sample, and the bioluminescence value was read for 10 seconds.

\section{Western blot analysis}

Total protein was extracted from cells using ice-cold RIPA buffer (Sigma, St. Louis, MO, USA) according to the manufacturer's protocol. Proteins were separated by $12 \%$ sodium dodecyl sulfate-polyacrylamide gel electrophoresis (SDS-PAGE) gels and transferred to polyvinylidene difluoride membranes (PVDF) (Sigma, St. Louis, MO, USA). The PVDF membranes were blocked with 5\% skim milk in Tris-buffered saline with Tween (TBST) at room temperature for $2 \mathrm{~h}$. Then, the membranes were placed into the primary antibody solution for incubation at $4{ }^{\circ} \mathrm{C}$ with gentle shaking overnight. The membranes were washed 3 times/5 min with TBST and placed into the secondary antibody solution for incubation at room temperature for $1 \mathrm{~h}$ and rewashed 3 times/5 min with TBST. The band development of protein was visualized using the ECL chemiluminescence reagent at a chemiluminescent detection system, and the grey values of target proteins were performed image analysis using Image $\mathrm{J}$ software. Antibodies used in this study are as follows: DNMT1 (1:1000), VEGF (1:1000), PI3K (1:1000), Akt (1:1000), Enos (1:1000), GAPDH (1:1000) and $\beta$-actin (1:1000). (all primary antibodies were purchased from Abcam, Cambridge, MA, USA). 


\section{Quantitative real-time polymerase chain reaction (RT-qPCR)}

Total RNA isolation from cells and tissues was performed using the Trizol reagent (Invitrogen, Carlsbad, CA, U.S.A.) according to the manufacturer's instructions. The RNA concentration was detected by NanoDrop2000 (Thermo Fisher Scientific Inc., Waltham, Massachusetts, USA) and stored at $-80^{\circ} \mathrm{C}$ for further use. According to gene sequences published in GenBank's database, primers were designed using Primer 5.0 design software and synthesized by Shanghai GenePharma Co., Ltd. (Shanghai, China). Reverse transcription was performed with approximately $0.5 \mu \mathrm{g}$ of total RNA into cDNA with the Superscript ${ }^{\text {TM }}$ reverse transcription system (Takara, Dalian, Japan). Aliquots of cDNA were used for PCR amplification with primer-specific for miR-148a-3p, DNMT1, VEGF and $\beta$-actin. RT-qPCR reaction was performed as follows: $95^{\circ} \mathrm{C}$ for $3 \mathrm{~min}, 35$ cycles of denaturation at $95^{\circ} \mathrm{C}$ for $35 \mathrm{sec}, 58^{\circ} \mathrm{C}$ for $45 \mathrm{sec}$, and $72^{\circ} \mathrm{C}$ for $30 \mathrm{sec}$ and $10 \mathrm{~min}$ at $72^{\circ} \mathrm{C}$ using SYBR Green PCR master mix reagents (Takara, Dalian, Japan) in the ABI StepOne Plus Real-time PCR system. The relative quantification of mRNA expression levels was calculated using the $2-\Delta \Delta C T$ method.

\section{Bisulfite-sequencing PCR (BSP)}

A total of $500 \mathrm{ng}$ genomic DNA was extracted from the tissues and cells and subjected to bisulfite conversion using the EZ DNA Methylation-Gold kit (Zymo Research, CA, USA). The VEGF promoter was amplified by PCR with Phusion U Hot Start DNA Polymerase (Thermo Fisher Scientific Inc.). Sanger sequencing was performed on the PCR products. Five single molecules were sequenced for each sample.

\section{Methylation-specific polymerase chain reaction (MSP)}

MSP was performed to detect methylated (M) or unmethylated $(U)$ alleles of the VEGF promoter CpG island. The PCR procedure was performed using Phusion U Hot Start DNA polymerase (Thermo Fisher Scientific Inc.): $98^{\circ} \mathrm{C}$ for $2 \mathrm{~min}$, followed by 35 cycles: $98^{\circ} \mathrm{C}$ for $15 \mathrm{~s}, 58^{\circ} \mathrm{C}$ for $30 \mathrm{~s}$ and $72{ }^{\circ} \mathrm{C}$ for $40 \mathrm{~s}$.

\section{Cell proliferation assay}

After transfection, the cells were cultured in 96 -well plates for $24 \mathrm{~h}$, and the cell proliferation was detected by cell counting kit-8 (CCK-8) (Dojindo Laboratories, Kumamoto, Japan). According to the manufacturer's instructions, $10 \mu \mathrm{L}$ of CCK-8 reagent was added to each well of cell plates. Then, the cells were incubated at $37^{\circ} \mathrm{C}$ for $3 \mathrm{~h}$. The absorbance was detected at $450 \mathrm{~nm}$ in each well using a multifunctional microplate reader (Thermo Fisher Scientific, Waltham, MA, USA). The above actions were repeated 3 times.

\section{Flow cytometry assays}

The cell apoptosis was detected using a FACS Aria Sorter (Becton Dickinson, San Jose, CA, USA). The transfected cells were collected, washed three times with PBS and resuspended in $500 \mu \mathrm{L}$ buffer solution (PBS with calcium), mixed with $5 \mu \mathrm{L}$ of Annexin-V-fluorescein isothiocyanate (FITC) and $2.5 \mu \mathrm{L}$ of propidium iodide $(\mathrm{PI})$, followed by incubation at $4^{\circ} \mathrm{C}$ for $30 \mathrm{~min}$. After $400 \mu \mathrm{L}$ staining buffer was added, the cells were immediately calculated and analyzed with flow cytometry (Becton, Dickinson and 
Company, Franklin Lakes, New Jersey, USA). Annexin V-positive cells were apoptotic cells, and the right upper quadrant and the right lower quadrant presented the apoptotic cells. Each experiment was conducted 3 times.

\section{Transwell invasion assay}

The invasive ability of pcDNA 3.1 transfected the cells was assessed using BD Falcon ${ }^{\mathrm{TM}}$ cell culture inserts coated with BD Matrigel ${ }^{\mathrm{TM}}$ Basement Membrane Matrix (BD Biosciences, Bedford, MA, USA). Transfected cells were resuspended at a density of $5 \times 10^{5}$ cells in serum-free medium and inoculated into the upper chamber of the assay system, while $0.5 \mathrm{~mL}$ of DMEM containing $10 \%$ FBS was added to the lower chamber. Following incubation for $48 \mathrm{~h}$, the invaded cells were washed 3 times with PBS, fixed with $4 \%$ paraformaldehyde solution (Affymetrix, Cleveland, OH, USA) for $15 \mathrm{~min}$ and stained with methyl violet $(0.01 \% \mathrm{v} / \mathrm{v}$; Guidechem, Shanghai, China) for $30 \mathrm{~min}$. The numbers of invaded cells were then counted under a light microscope (IX73-F22FL/PH; Olympus America, Inc., Melville, NY, USA). Each experiment was conducted 3 times.

\section{Determination of NO concentration}

The cells were cultured for $48 \mathrm{~h}$ in colorless DMEM. Nitric oxide (NO) quantified by the accumulation of nitrite (a stable breakdown product of nitric oxide) in the medium. $500 \mu \mathrm{L}$ of the cell culture supernatant was taken as a sample and incubated with an equal volume $(500 \mu \mathrm{L})$ of Griess reagent for $10 \mathrm{~min}$ at room temperature, and the absorbance was measured by spectrophotometry at $560 \mathrm{~nm}$. The sodium nitrite $\left(\mathrm{NaNO}_{2}\right)$ dissolved in colorless DMEM was used as a standard. According to the manufacturer's instructions, the amount of nitrite (a stable metabolite of NO) was assessed in the cell culture supernatant using a Griess reagent kit (Promega, Madison, WI).

\section{Statistical analysis}

The data are shown as the mean \pm standard error of the mean (SEM) of three or more independent experiments were performed. Statistical differences were evaluated using a one-way ANOVA, two-way ANOVA and the t-test. $P$-values $<0.05$ were considered to indicate statistically significant differences. Statistics were performed with SPSS Statistics version 20.0 software.

\section{Results}

\section{The expression of miR-148a-3p was significantly up-regulated in glioma}

To investigate the role of miR-148a-3p in the progression of glioma, we first analyzed the expression level of miR-148a-3p in 20 pairs of glioma tissues and their corresponding healthy tissues. The RT-qPCR analysis revealed that the expression of miR-148a-3p was significantly up-regulated in glioma (Fig. $1 \mathrm{~A}$ ). We further analyzed the relative expression of miR 148a-3p in HEB and U87 cells. U87 cell lines had a 
relatively higher expression of miR-148a-3p compared with the HEB cell lines (Fig. 1B). These findings suggested that the up-regulation of miR-148a-3p may be involved in glioma development.

\section{MiR-148a-3p promotes glioma cell proliferation and invasion}

We next assessed the functional biological role of miR-148a-3p in glioma U87 cells by determining the effects of miRNA overexpression and promotion on cell proliferation and invasion using the CCK-8 assay and Transwell assays, respectively. The successful overexpression of mature miR-148a-3p in U87 cells was confirmed by RT-qPCR (Fig. 2A). For the CCK-8 assay and Transwell assay, overexpression of miR148a resulted in a higher proliferation in U87 cells as compared with controls (Fig. 2B). Similarly, overexpression of miR-148a-3p led to a significant promotion of invasion in U87 cells (Fig. 2C). The above results show that miR-148a-3p promotes proliferation and invasion in glioma cells.

\section{miR-148a-3p downregulates DNMT1 expression by directly targeting its 3'-UTR.}

To explore downstream targets of miR-148a-3p, TargetScan was used to predict its targets, and the results showed that miR-148a-3p might directly target to DNMT1 through a potential binding site in the 3'UTR of DNMT1 at the position 48-55 (Fig. 3A ). To verify whether miR-148a-3p could bind to 3'-UTR of DNMT1, we cloned the 3'-UTR of the DNMT1gene downstream of the luciferase promoter and performed luciferase activity assays. Luciferase activity was significantly decreased in the wild type cells and was not obviously altered in the mutant cells compared with control (Fig. 3B). Additionally, we detected whether the expression of DNMT1 is regulated by miR-148a-3p. In U87 cells, the overexpression of miR-148a-3p reduced the expression of DNMT1 at the protein and mRNA levels (Fig. 3C and D).

\section{VEGF shows high expression and low methylation in glioma}

CpG islands in the promoter region of the VEGF gene were analyzed with 2000 bp nucleotide sequences upstream of the VEGF gene promoter using MethPrimer software. The results showed that the CpG islands were present in the promoter region of the VEGF gene (Fig. 4A), indicating that the expression of the VEGF gene was affected by promoter methylation. To verify that the expression of the VEGF gene could be affected by promoter methylation, we used MSP to detect the methylation levels of CpG islands in the promoter region of VEGF. We found that the VEGF gene promoter region was hypermethylated in healthy tissues, and methylation of the VEGF gene promoter region was significantly down-regulated in glioma tissues (Fig 4B and C). Detection of VEGF expression showed that the VEGF expression in glioma exhibited high mRNA and protein levels, whereas that in healthy tissues showed low expression levels(Fig $4 \mathrm{D}$ and $\mathrm{E}$ ), which suggests that VEGF expression was negatively correlated with VEGF methylation rate. Overall, VEGF was found to exhibit high expression and low methylation in glioma.

\section{Overexpression of VEGF promotes the glioma cell proliferation, invasion and inhibited cell apoptosis}

Overexpression of VEGF was performed by transfection of pcDNA-VEGF. The results showed that the mRNA and protein levels of VEGF were significantly increased after the cells were transfected of pcDNAVEGF (Fig. 5A and B). We investigated the role of VEGF in cell proliferation, invasion and apoptosis. Cell 
proliferation was detected by CCK-8, and the results showed that VEGF overexpression resulted in a significant promoted in cell proliferation (Fig. 6C). Flow cytometry assay results showed that overexpression of VEGF significantly inhibited cell apoptosis after $48 \mathrm{~h}$ of transfection (Fig. 6D). The invasive ability of cells plays a vital role in the development of tumors. We evaluated the invasive ability of cells by the Transwell assay. Compared with control, the capacity of cell invasive was significantly increased after the overexpression of VEGF (Fig. 6E).

\section{MiR-148a-3p up-regulates the expression levels of VEGF by directly targeting DNMT1}

The miR-148a-3p gain of function in U87 cells was investigated to determine whether miR-148a-3p was involved in the regulation of VEGF expression. qRT-PCR showed that the mRNA and protein expression of VEGF was significantly elevated when miR-148a-3p was overexpression (Fig. 6A and B). miR-148a-3p mimic contributed to the reduced methylation level of VEGF in U87 cells of glioma (Fig. 6C). In addition, we also found that the expression level of DNMT1 was significantly decreased after mir-148a-3p overexpression (Fig. 3D and E). Therefore, we speculated that there exists a specific relationship between the low methylation level of VEGF and the inhibition of DNMT1 by miR-148a-3p.

To confirm our hypothesis, we overexpressed DNMT1 in U87 cells, and the results showed that the expression of VEGF was significantly reduced (Fig. 6E and F) and the methylation level was increased (Fig. 6G) when DNMT1 was overexpression, as opposed to that of si-DNMT1-treated U87 cells. MiR-148a$3 p$ levels were verified by qRT-PCR (Fig. 6D and F). The above results show that miR-148a-3p promotes cell growth and inhibits cell death in glioblastoma. The methylation level of VEGF is negatively regulated by miR-148a-3p, and miR-148a-3p inhibits VEGF expression by targeting DNMT1.

\section{Silencing VEGF inhibits the activation of the PI3K/Akt/eNOS pathway and induces apoptosis of glioma cells}

As more and more evidence shown that the PI3K/Akt/eNOS pathway plays a vital role in the development of tumors, we investigated whether VEGF regulates glioma through the PI3K/Akt/eNOS pathway. Silencing VEGF was performed by transfection of si-VEGF to U87 cells. The mRNA and protein levels of VEGF were significantly reduced after si-VEGF transfected (Fig. 7A, B and C). As shown in Fig. 7D, the phosphorylation levels of PI3K (Fig. 7E), Akt (Fig. 7F) and eNOS (Fig. 7G) are significantly reduced after VEGF was knockdown. In addition, we also measured the nitric oxide production, and the results showed that the production of nitric oxide was reduced considerably in U87 cells treated with si-VEGF (Fig. $7 \mathrm{H})$. Then, we investigated the proliferation (Fig. 7I), invasion (Fig. 7J) and apoptosis of U87 cells, and found that the apoptotic cells significantly increased (Fig. 7K), the spread and infiltration of the cells were inhibited after the cells were treated with si-VEGF, and this change is suppressed after treatment with 740 Y-P. These results indicate that silencing VEGF was induced apoptosis and inhibited proliferation, invasion of glioma cells by the PI3K/Akt/eNOS pathway.

\section{Discussion}


Glioma is the most common form of adult brain tumors with intense immersion and poor prognosis. The high lethality rate of gliomas highlights the urgent need for efficient treatment. The pathogenesis of glioma is complex and can be induced by a variety of mechanisms, as shown by abnormal activation of glioma stem cells[28], tumor angiogenesis[29] and isocitrate dehydrogenase mutation[30]. VEGF acts as a regulator of angiogenesis has been widely recognized as a critical contributor to the development and progression of glioma $[12,14]$. Our study shows that VEGF is up-regulated and hypomethylated in glioma. VEGF silencing, the methylation level of VEGF was significantly increased, and the cell proliferation and invasion of glioma cells were inhibited. Our results indicate for the first time that the methylation of VEGF plays a vital role in inhibiting the development of glioma.

The growth and metastasis of cancer cells depend on angiogenesis. The vascular endothelial growth factor is the most potent pro-angiogenic factor[15, 22]. It is involved in the regulation of cell proliferation, survival and invasiveness[14]. It has been reported that the expression of VEGF was increased in various tumor tissues, and silencing it can significantly improve the physiological and pathological changes of tumors and inhibit tumor progression[11, 12,14]. Autenshlyus Al et al. has reported that the expression of VEGF was significantly up-regulated in invasive ductal cancer and fibroadenoma of the breast[31]. Huang D et al.'s research found that VEGF was increased observably in nasopharyngeal carcinoma, compared with healthy tissues. Consistent with the report, our study showed that the mRNA and protein levels of VEGF were significantly elevated in the glioma compared with healthy tissues. Overexpression VEGF promoted the proliferation and invasion of U87 cells and accelerated the deterioration of glioma. Silencing VEGF was performed by transfection of siRNA of VEGF to U87 cells, which induce apoptosis and inhibit cell proliferation and invasion. In addition, we also found that the promoter methylation of VEGF was significantly reduced in glioma compared with healthy tissues. Overexpression VEGF, the methylation level of VEGF was increased, and the expression level was down-regulated, which inhibits the proliferation and invasion of the U87 cell. Our findings confirm previous reports that the expression of VEGF is regulated by promoter methylation, and the methylation of VEGF is involved in the development of tumors[16]. Da MX et al. was research found that DNA hypomethylation may activate VEGF transcription and promote carcinogenesis and the progression of gastric cancer. Hypermethylation of VEGF and VEGF silencing can inhibit the carcinogenic effect of VEGF and the progress of gastric cancer[15]. Cui $S$ et al. has reported that DNA Methylation levels of VEGF in atherosclerotic were upregulated, and folic acid supplementation delays atherosclerotic lesion development by modulating VEGF DNA methylation levels in vivo and vitro[17]. These studies are consistent with our results, further confirming our hypothesis that VEGF methylation plays a vital role in the occurrence and development of cancer, and we can improve disease by increasing the methylation level of VEGF.

As a current research hotspot, miRNA has attracted more and more attention[20]. MiR-148a-3p is one of the new miRNAs has been found that has attracted considerable interest since it is implicated many types of cancer[24,25]. miR-148a inhibits early relapsed colorectal cancers and the secretion of VEGF by indirectly targeting HIF-1a under non-hypoxia/hypoxia conditions[32], and it promotes proliferation of bladder cancer T24 cells and inhibits apoptosis of the bladder cancer[33]. miR-148a-3p has also been widely reported in glioma, for example, miR-148a-3p is a prognostic oncomiR, and it was up-regulated in 
glioma, which targets MIG6 and BIM to regulate apoptosis in glioblastoma[34]; miR-148a promotes proliferation and metastasis of glioblastoma via targeting CADM1-miR-148a effect on glioma cells by CADM1[27]; miR-148a was induced by NF-KB and sustained TGF- $\beta$ /Smad signaling activation in glioblastoma[35]; miR-148a increases glioma cell migration and invasion by down-regulating GADD45A in human gliomas with IDH1 R132H mutations[36]. In addition to these reports, it has been reported that miR-148a-3p-regulates gene expression through DNMT1-mediated methylation, thereby inhibits the occurrence and development of cancer. Shi HJ et al. research showed that miR-148a suppresses cell invasion and migration in gastric cancer by targeting DNA methyltransferase 1[37]. Xu et al. reported that miRNA-148a regulates the expression of the estrogen receptor through DNMT1-mediated DNA methylation in breast cancer cells[26]. In this study, we investigated the interaction between miR-148a-3p and VEGF found that miR-148a-3p promotes the expression of VEGF by inhibiting DNMT1-mediated methylation of VEGF and promotes proliferation and invasion of glioma cells. Proliferation and invasion of glioma cells were inhibited, and apoptosis was induced when DNMT1 is overexpressed or VEGF is silenced. Those studies support our hypothesis that miR-148a-3p regulates the promoter methylation of VEGF and further regulates the progression of glioma.

The previous study showed that the PI3K/AKK/eNOS pathway is involved in various pathogenetic processes[38-40]. Wang et al. have reported that overexpression of osteopontin induces angiogenesis of endothelial progenitor cells via the av- $33 / \mathrm{PI} 3 \mathrm{~K} / \mathrm{Akt} / \mathrm{eNOS} / \mathrm{NO}$ signaling pathway in glioma cells[21], which indicated that activation of the PI3K / AKK / eNOS pathway promotes the development of glioma. In our study, si-VEGF and $740 \mathrm{Y}-\mathrm{P}$ was used to interfere with the treatment of cells. Phosphorylation of related protein $\mathrm{PI} 3 \mathrm{~K}$, Akt, eNOS of the PI3K/AKK/eNOS pathway was reduced after VEGF was silenced. Glioma cell apoptosis was induced, proliferation and invasion were inhibited, and eNOS are significantly reduced after VEGF was silenced. The opposite is exact for cells treated with $740 \mathrm{y}-\mathrm{p}$. Those changes were suppressed by the treatment of the cells with 740 Y-P and si-VEGF. Our results showed that VEGF regulated glioma cells function through the PI3K/Akt/eNOS pathway.

\section{Conclusion}

In summary, miR-148a-3p inhibits DNMT1-mediated methylation of VEGF and promotes proliferation and invasion in glioma cells via the PI3K/Akt/eNOS signaling pathway.

\section{Declarations}

\section{Competing interests}

The authors declare that there are no competing interests.

\section{Abbreviations}

VEGF, vascular endothelial growth factor 
DNMT1, DNA methyltransferase 1

FBS, fetal bovine serum

Ethics approval and consent to participate

The study was approved by the Ethics Committee for clinical research in the Huaihe Hospital of Henan University.

\section{Consent for publication}

Not applicable.

\section{Availability of data and material}

Not applicable.

\section{Authors' contributions}

Jun-feng Huo and Xiao-bing Chen performed the experiments. Jun-feng Huo and Xiao-bing Chen wrote the manuscript.

\section{Acknowledgment}

None.

\section{Funding}

None.

\section{References}

1. Ostrom QT, Gittleman H, Liao P, Vecchione-Koval T, Wolinsky Y, Kruchko C, Barnholtz-Sloan JS. CBTRUS Statistical Report: Primary brain and other central nervous system tumors diagnosed in the United States in 2010-2014. Neuro Oncol 2017, 19(5):v1-v88.

2. Jiao J, Zhang R, Li Z, Yin Y, Fang X, Ding $X$ et al. Nuclear Smad6 promotes gliomagenesis by negatively regulating PIAS3-mediated STAT3 inhibition. Nat Commun 2018, 9(1):2504.

3. Norden AD, Drappatz J, Wen PY. Antiangiogenic therapies for high-grade glioma. Nat Rev Neurol 2009, 5(11):610-620.

4. Jiang L, Lin C, Song L, Wu J, Chen B, Ying Z et al. MicroRNA-30e* promotes human glioma cell invasiveness in an orthotopic xenotransplantation model by disrupting the NF-kappaB/lkappaBalpha negative feedback loop. J Clin Invest 2012, 122(1):33-47.

5. Hoelzinger DB, Demuth T, Berens ME. Autocrine factors that sustain glioma invasion and paracrine biology in the brain microenvironment. J Natl Cancer Inst 2007, 99(21):1583-1593. 
6. Li T, Kang G, Wang T, Huang $\mathrm{H}$. Tumor angiogenesis and anti-angiogenic gene therapy for cancer. Oncol Lett 2018, 16(1):687-702.

7. Fantin A, Ruhrberg C. The Embryonic Mouse Hindbrain and Postnatal Retina as In Vivo Models to Study Angiogenesis. Methods in molecular biology (Clifton, NJ) 2015, 1332:177-188.

8. Pu W, He L, Han X, Tian X, Li Y, Zhang H et al. Genetic Targeting of Organ-Specific Blood Vessels. Circulation research 2018, 123(1):86-99.

9. Shang T, Li S, Zhang Y, Lu L, Cui L, Guo FF. Hypoxia promotes differentiation of adipose-derived stem cells into endothelial cells through demethylation of ephrinB2. Stem cell research \& therapy 2019 , 10(1):133.

10. Kumanishi S, Yamanegi K, Nishiura H, Fujihara Y, Kobayashi K, Nakasho K, Futani H, Yoshiya S. Epigenetic modulators hydralazine and sodium valproate act synergistically in VEGI-mediated antiangiogenesis and VEGF interference in human osteosarcoma and vascular endothelial cells. Int $\mathrm{J}$ Oncol 2019, 55(1):167-178.

11. Di Y, Chen XL. Inhibition of LY294002 in retinal neovascularization via down-regulation the PI3K/AKTVEGF pathway in vivo and in vitro. Int J Ophthalmol 2018, 11(8):1284-1289.

12. Chen HX, Xu XX, Tan BZ, Zhang Z, Zhou XD. MicroRNA-29b Inhibits Angiogenesis by Targeting VEGFA through the MAPK/ERK and PI3K/Akt Signaling Pathways in Endometrial Carcinoma. Cell Physiol Biochem 2017, 41(3):933-946.

13. Zhao J, Zhang ZR, Zhao N, Ma BA, Fan QY. VEGF Silencing Inhibits Human Osteosarcoma Angiogenesis and Promotes Cell Apoptosis via PI3K/AKT Signaling Pathway. Cell Biochem Biophys 2015, 73(2):519-525.

14. Zhou Y, Li S, Li J, Wang D, Li Q. Effect of microRNA-135a on Cell Proliferation, Migration, Invasion, Apoptosis and Tumor Angiogenesis Through the IGF-1/PI3K/Akt Signaling Pathway in Non-Small Cell Lung Cancer. Cell Physiol Biochem 2017, 42(4):1431-1446.

15. Da MX, Zhang YB, Yao JB, Duan YX. DNA methylation regulates expression of VEGF-C, and Sadenosylmethionine is effective for VEGF-C methylation and for inhibiting cancer growth. Brazilian Journal of Medical and Biological Research 2014, 47(12):1021-1028.

16. Pisani F, Cammalleri M, Dal Monte M, Locri F, Mola MG, Nicchia GP, Frigeri A, Bagnoli P, Svelto M. Potential role of the methylation of VEGF gene promoter in response to hypoxia in oxygen-induced retinopathy: beneficial effect of the absence of AQP4. J Cell Mol Med 2018, 22(1):613-627.

17. Cui S, Li W, Lv X, Wang P, Gao Y, Huang G. Folic Acid Supplementation Delays Atherosclerotic Lesion Development by Modulating MCP1 and VEGF DNA Methylation Levels In Vivo and In Vitro. Int J Mol Sci 2017, 18(5).

18. Sun Z, Li Y, Wang Y, Fan X, Xu K, Wang K et al. Radiogenomic analysis of vascular endothelial growth factor in patients with diffuse gliomas. Cancer imaging : the official publication of the International Cancer Imaging Society 2019, 19(1):68.

19. Jiang Y, Zhou J, Zou D, Hou D, Zhang H, Zhao J et al. Overexpression of Limb-Bud and Heart (LBH) promotes angiogenesis in human glioma via VEGFA-mediated ERK signalling under hypoxia. 
EBioMedicine 2019, 48:36-48.

20. Acunzo M, Romano G, Wernicke D, Croce CM. MicroRNA and cancer-a brief overview. Adv Biol Regul 2015, 57:1-9.

21. Wang Y, Yan W, Lu X, Qian C, Zhang J, Li P et al. Overexpression of osteopontin induces angiogenesis of endothelial progenitor cells via the avbeta3/PI3K/AKT/eNOS/NO signaling pathway in glioma cells. Eur J Cell Biol 2011, 90(8):642-648.

22. Huang Y, Rajappa P, Hu W, Hoffman C, Cisse B, Kim JH et al. A proangiogenic signaling axis in myeloid cells promotes malignant progression of glioma. J Clin Invest 2017, 127(5):1826-1838.

23. Kim S, Choi JY, Seok HJ, Park MJ, Chung HY, Bae IH. miR-340-5p Suppresses Aggressiveness in Glioblastoma Multiforme by Targeting Bcl-w and Sox2. Mol Ther Nucleic Acids 2019, 17:245-255.

24. Xiong Q, Wu S, Wang J, Zeng X, Chen J, Wei M et al. Hepatitis B virus promotes cancer cell migration by downregulating miR-340-5p expression to induce STAT3 overexpression. Cell Biosci 2017, 7:16.

25. Zhao P, Ma W, Hu Z, Zhang Y, Zhang S, Wang Y. Up-regulation of miR-340-5p promotes progression of thyroid cancer by inhibiting BMP4. J Endocrinol Invest 2018, 41(10):1165-1172.

26. Xu Y, Chao L, Wang J, Sun Y. miRNA-148a regulates the expression of the estrogen receptor through DNMT1-mediated DNA methylation in breast cancer cells. Oncol Lett 2017, 14(4):4736-4740.

27. Cai Q, Zhu A, Gong L. Exosomes of glioma cells deliver miR-148a to promote proliferation and metastasis of glioblastoma via targeting CADM1. Bull Cancer 2018, 105(7-8):643-651.

28. Rajakulendran N, Rowland KJ, Selvadurai HJ, Ahmadi M, Park NI, Naumenko S et al. Wnt and Notch signaling govern self-renewal and differentiation in a subset of human glioblastoma stem cells. Genes Dev 2019, 33(9-10):498-510.

29. Chen Y, Wang D, Peng H, Chen X, Han X, Yu J et al. Epigenetically upregulated oncoprotein PLCE1 drives esophageal carcinoma angiogenesis and proliferation via activating the PI-PLCepsilon-NFkappaB signaling pathway and VEGF-C/ Bcl-2 expression. Mol Cancer 2019, 18(1):1.

30. Christensen BC, Smith AA, Zheng S, Koestler DC, Houseman EA, Marsit CJ et al. DNA methylation, isocitrate dehydrogenase mutation, and survival in glioma. J Natl Cancer Inst 2011, 103(2):143-153.

31. Autenshlyus Al, Arkhipov SA, Kunts TA, Marinkin IO, Mikhailova ES, Karpukhina XV, Varaksin NA. Cytokine profiles of tumor supernatants in invasive ductal cancer and fibroadenoma of the breast and its relationship with VEGF-A expression in the tumors. International journal of immunopathology and pharmacology 2017, 30(1):83-88.

32. Tsai HL, Miao ZF, Chen YT, Huang CW, Yeh YS, Yang IP, Wang JY. miR-148a inhibits early relapsed colorectal cancers and the secretion of VEGF by indirectly targeting HIF-1alpha under nonhypoxia/hypoxia conditions. J Cell Mol Med 2019, 23(5):3572-3582.

33. Xiang M, Yuan W, Zhang W, Huang J. Expression of miR-490-5p, miR-148a-3p and miR-608 in bladder cancer and their effects on the biological characteristics of bladder cancer cells. Oncol Lett 2019, 17(5):4437-4442. 
34. Kim J, Zhang Y, Skalski M, Hayes J, Kefas B, Schiff D et al. microRNA-148a is a prognostic oncomiR that targets MIG6 and BIM to regulate EGFR and apoptosis in glioblastoma. Cancer Res 2014, 74(5):1541-1553.

35. Wang H, Pan JQ, Luo L, Ning XJ, Ye ZP, Yu Z, Li WS. NF-kappaB induces miR-148a to sustain TGFbeta/Smad signaling activation in glioblastoma. Mol Cancer 2015, 14:2.

36. Cui D, Sajan P, Shi J, Shen Y, Wang K, Deng X, Zhou L, Hu P, Gao L. MiR-148a increases glioma cell migration and invasion by downregulating GADD45A in human gliomas with IDH1 R132H mutations. Oncotarget 2017, 8(15):25345-25361.

37. Shi $H$, Chen $X$, Jiang $H$, Wang $X, Y u H$, Sun $P$, Sui $X$. miR-148a suppresses cell invasion and migration in gastric cancer by targeting DNA methyltransferase 1. Oncol Lett 2018, 15(4):4944-4950.

38. Chen L, Qin L, Liu X, Meng X. CTRP3 Alleviates Ox-LDL-Induced Inflammatory Response and Endothelial Dysfunction in Mouse Aortic Endothelial Cells by Activating the PI3K/Akt/eNOS Pathway. Inflammation 2019, 42(4):1350-1359.

39. Carrizzo A, Conte GM, Sommella E, Damato A, Ambrosio M, Sala M et al. Novel Potent Decameric Peptide of Spirulina platensis Reduces Blood Pressure Levels Through a PI3K/AKT/eNOS-Dependent Mechanism. Hypertension 2019, 73(2):449-457.

40. Yang HH, Chen Y, Gao CY, Cui ZT, Yao JM. Protective Effects of MicroRNA-126 on Human Cardiac Microvascular Endothelial Cells Against Hypoxia/Reoxygenation-Induced Injury and Inflammatory Response by Activating PI3K/Akt/eNOS Signaling Pathway. Cell Physiol Biochem 2017, 42(2):506518.

\section{Figures}

A



B

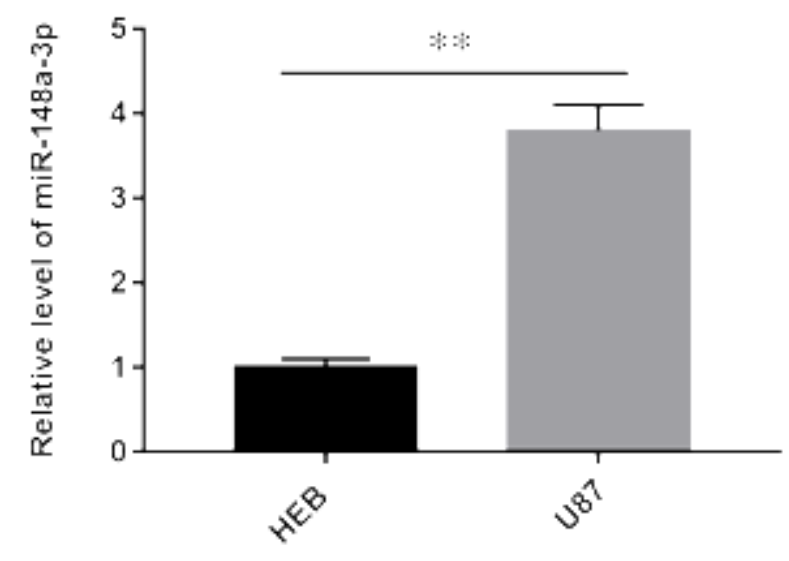

\section{Figure 1}


The expression of miR-148a-3p in glioma tissues and cell lines. Glioma tumor tissues and adjacent nonneoplastic tissues were collected to determine the expression level of miRNA-148a-3p (A). The miRNA1148a-3p levels in the human normal glial cell lines of HEB and the human glioma U87 cell lines were evaluated with RT-qPCR (B). * $P<0.05$, ** $P<0.01$ compared with the normal groups or control groups.

A

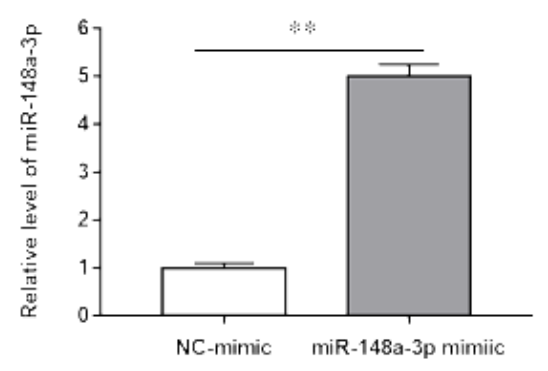

B

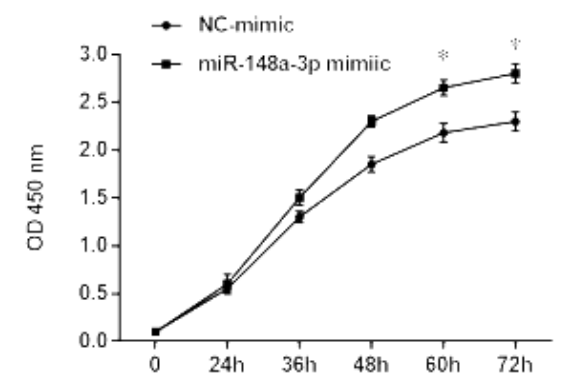

C

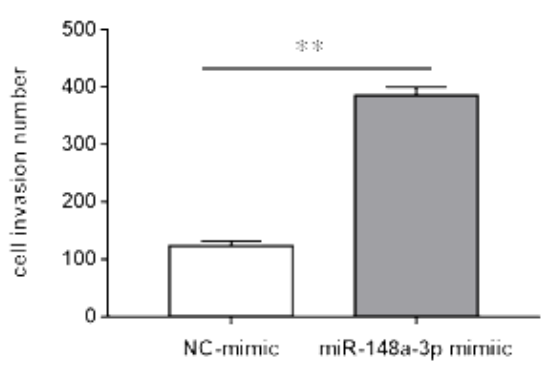

\section{Figure 2}

Overexpression of miR-148a-3p promotes glioma cell proliferation and invasion. U87 cells were transfected with the miR-148a-3p mimic $10 \mathrm{nmol} / \mathrm{L}$ or NC-mimic for $24 \mathrm{~h}$, and cells were collected for the biological functional analysis. The overexpression of miR-148a-3p was detected with RT-qPCR (A). The CCK-8 assay was used to identify the proliferation of U87 cells (B), and the Transwell invasion assay was used to analyze the invasion of U87 cells $(C) .{ }^{*} P<0.05, * \star P<0.01$.

A

DNMT1 3'UTR region $48-55$

miR-148a-3p

3' UGUUUCAAGACAUCACGUGACU 5'

||||||

DNMT1 wild type 3'UTR 5' ...CAGGAAUCCCCAACAUGCACUGA ...3'

DNMT1 wild type 3'UTR 5 '...CAGGAAUCCCCAACACAAUACCA....3'

B

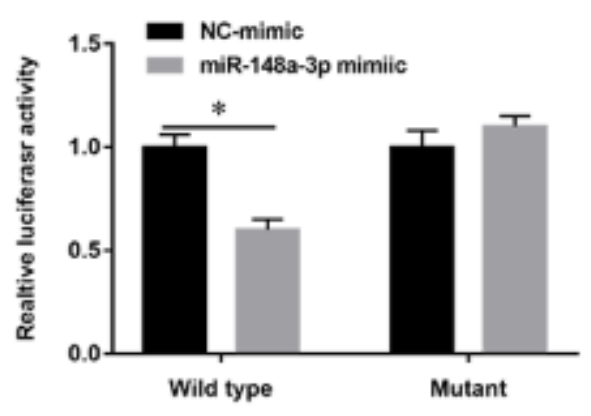

D
C
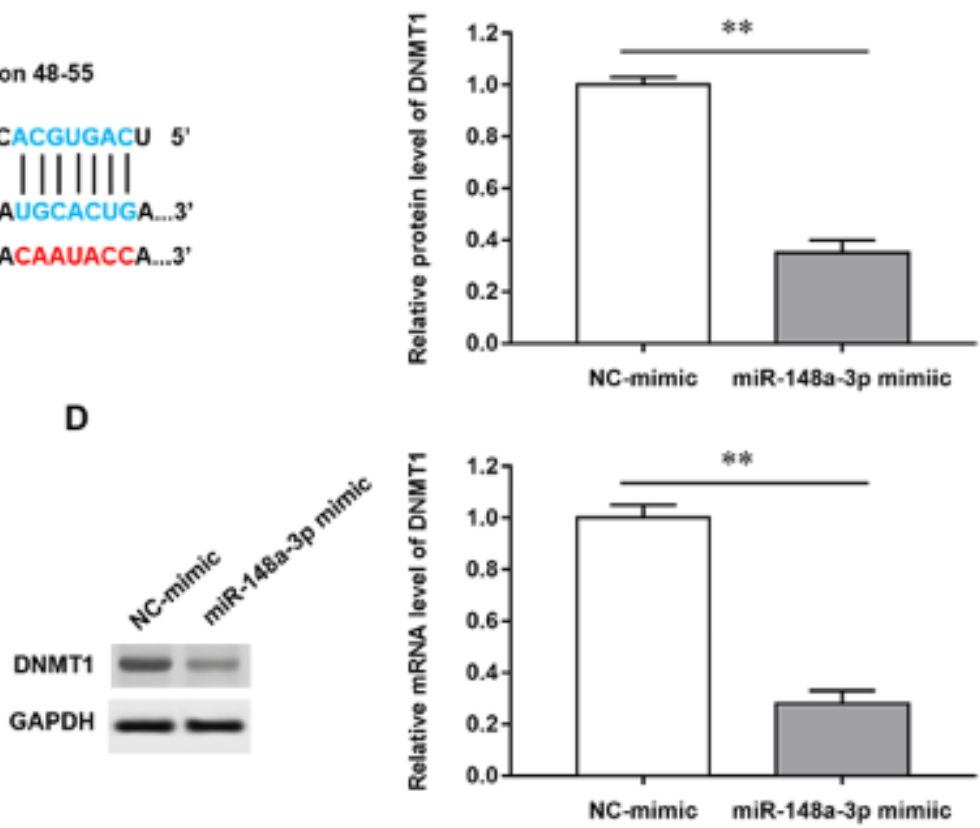

Figure 3 
MiR-148a-3p downregulates DNMT1 expression by directly targeting its 3'-UTR. The putative and corresponding mutant binding site of miR-148a-3p in the DNMT1 3'-UTR (A). A dual-luciferase reporter vector containing either wild type or mutated DNMT1 3'-UTR was co-transfected with miR-148a-3p precursor or scramble control in HEK293T cells, and the luciferase assay was performed (B). In U87 cells transfected with miR-148a-3p mimic, DNMT1 mRNA (C) and protein (D) expression levels were decreased. GAPDH was used as an internal control. ${ }^{*} \mathrm{P}<0.05$, $* * \mathrm{P}<0.01$ compared with the control groups.

A

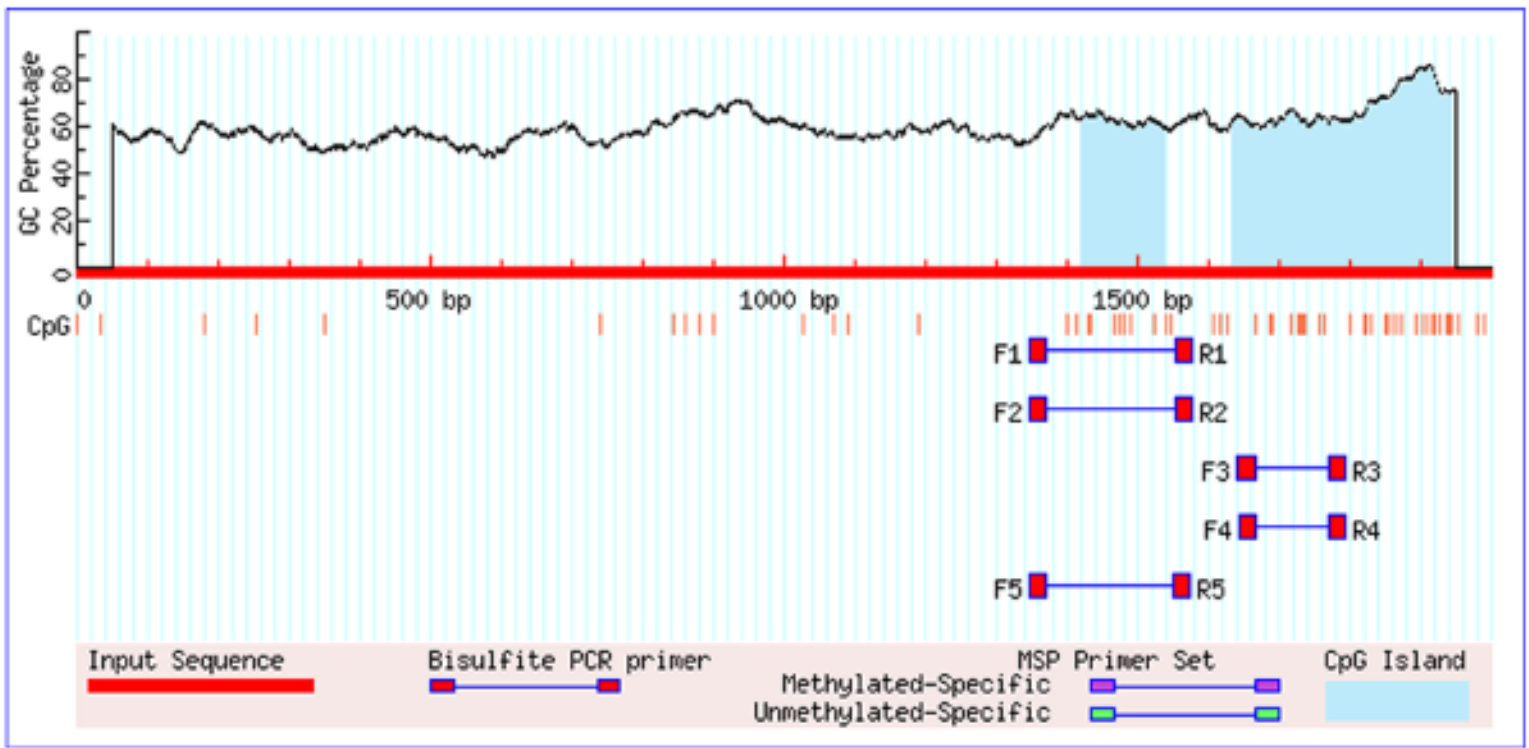

B

$$
\text { U M }
$$

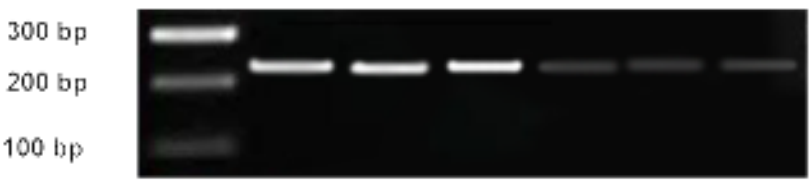

D



C
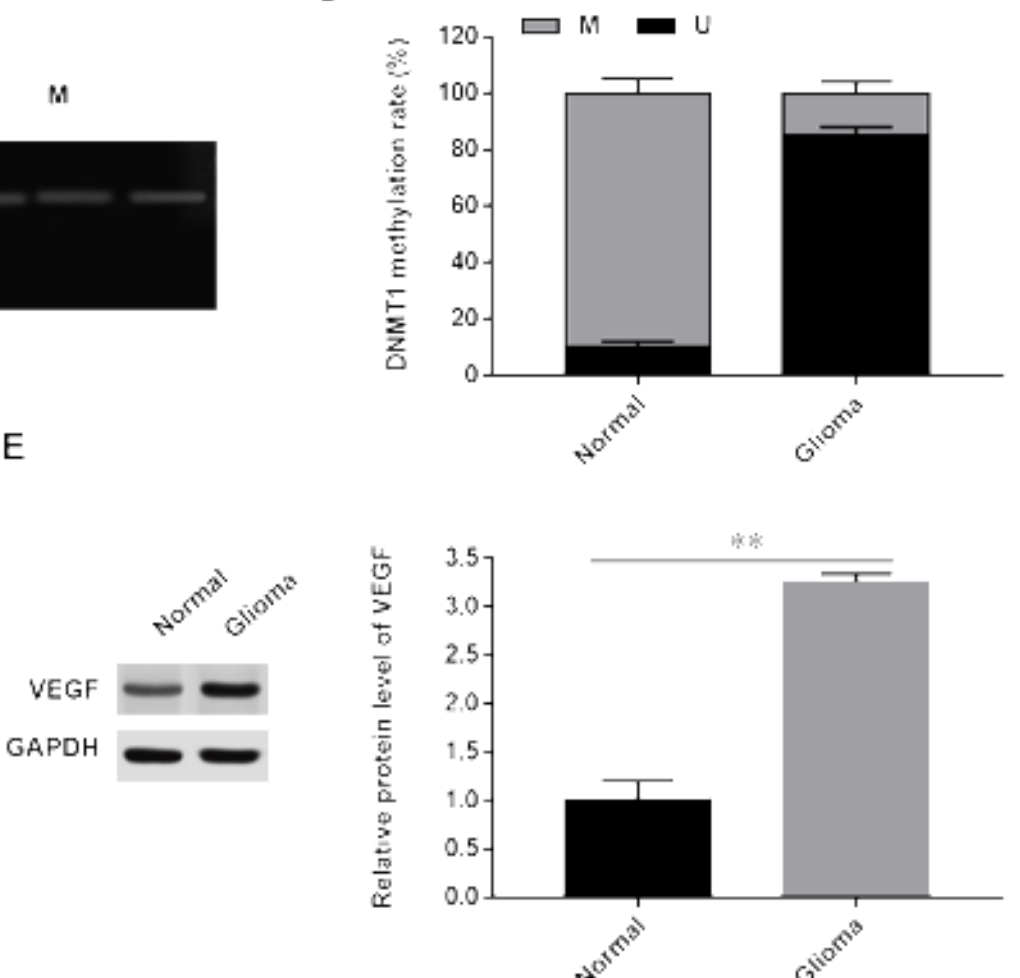






\section{Figure 4}

VEGF shows high expression and low methylation in glioma. MethPrimer predicts the distribution of CpG islands in the promoter region of the VEGF gene (A). The relationship between VEGF expression and VEGF methylation detected by BSP. Electrophoresis map of the methylation level of the VEGF promoter region in myocardial microvascular endothelium of glioma and healthy tissues detected by MSP (B). The mRNA (C) and protein (D) levels of VEGF in glioma and healthy tissues were detected with western blot and RT-qPCR. GC, guanine/cytosine; M, methylation; MF, methylated forward primer; MR, methylated reverse primer; $\mathrm{U}$, unmethylation; UF, unmethylated forward primer; UR, unmethylated Reverse primer. * $\mathrm{P}<$ 0.05 , ** $\mathrm{P}<0.01$ compared with the control groups.

A

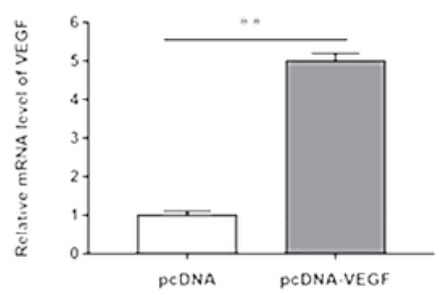

D

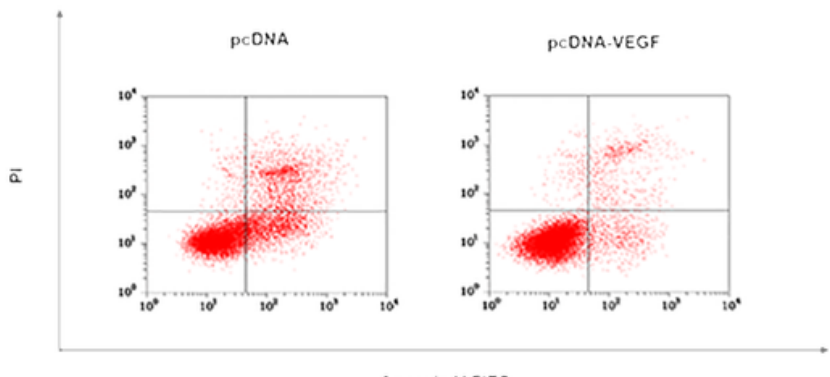

B
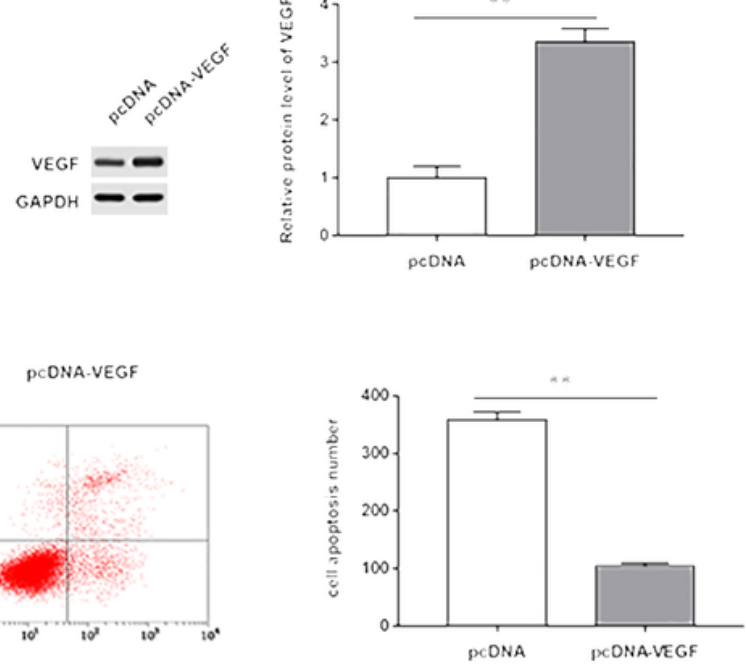

C

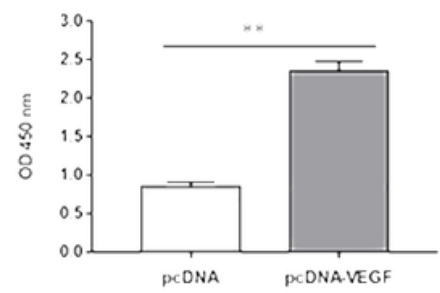

$E$

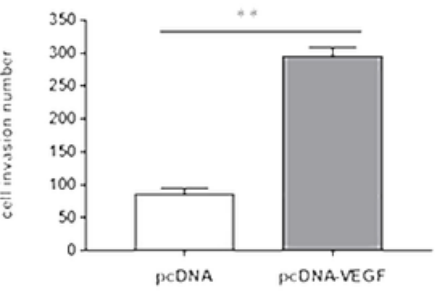

\section{Figure 5}

Overexpression of VEGF promotes glioma cell proliferation, invasion and inhibited cell apoptosis. U87 cells were transfected with pcDNA-VEGF $(2 \mu \mathrm{g} / \mathrm{mL})$ and cultured for $48 \mathrm{~h}$. Cells were collected for expression analysis and biological functional analysis. The mRNA $(A)$ and protein (B) levels of VEGF after transfection with pcDNA-VEGF were detected by Western blot in U87 cells. At $48 \mathrm{~h}$ after VEGF overexpression, the proliferation of U87 cells was determined by CCK-8 (C); cell apoptosis was evaluated by flow cytometry (D), and cell invasive ability was detected by Transwell assay (E). ${ }^{\star} P<0.05,{ }^{\star} * P<0.01$ compared with the control groups treated with pcDNA. 
A
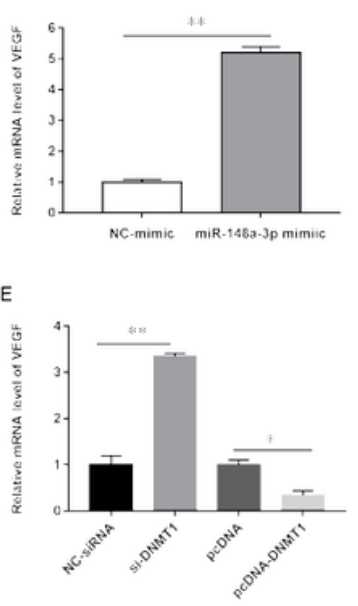
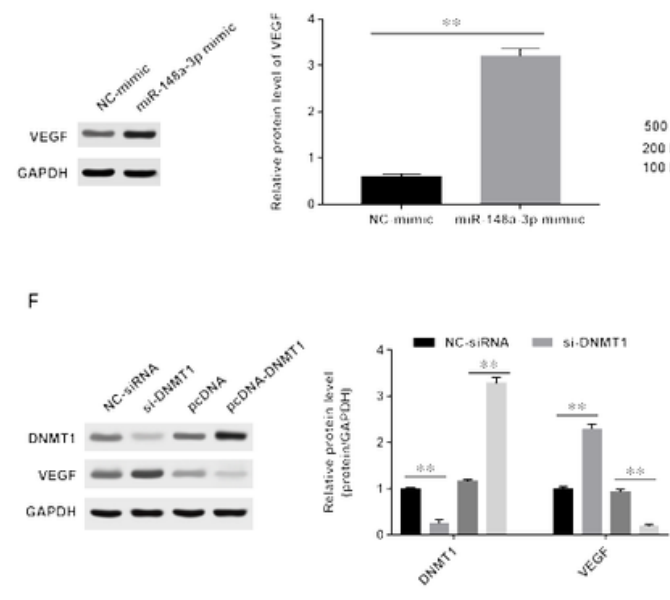

$500 \mathrm{bp}$

$100 \mathrm{bp}$
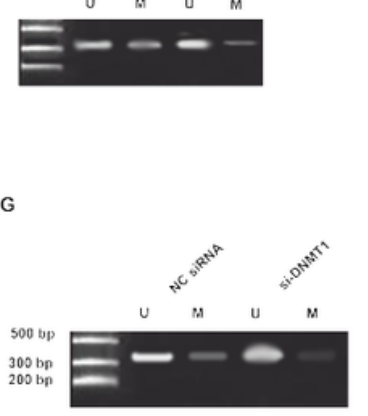

D
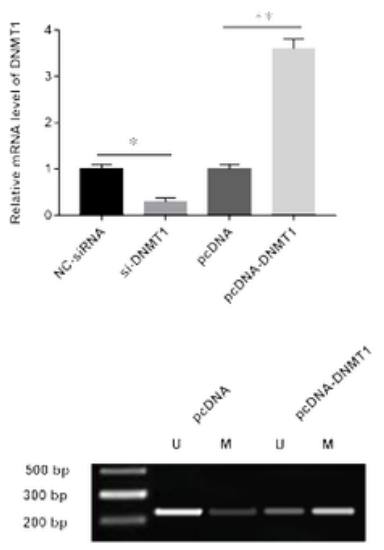

Figure 6

MiR-148a-3p up-regulates the expression levels of VEGF by directly targeting DNMT1. U87 cells were transfected with miR-148a-3p mimic (10 nmol/L) for $24 \mathrm{~h}$. The mRNA (A) and protein (B) expression of VEGF in U87 cells were detected with western blot and RT-qPCR, and the methylation status of VEGF in U87 cells was detected with bisulfite Sanger sequencing (C). DNMT1 silencing and overexpression were performed by transfection of si-DNMT1 $(10 \mathrm{nmol} / \mathrm{L})$ and pcDNA-DNMT1 $(2 \mu \mathrm{g} / \mathrm{mL})$ with U87 cells, respectively. U87 cells were transfected for $24 \mathrm{~h}$. RT-qPCR was used to assay the mRNA levels of DNMT1 (D) and VEGF (E), and western blot was used to quantify the protein levels of DNMT1 and VEGF (F). After DNMT1 knockdown or overexpression, the methylation level of VEGF in U87 cells was detected with BSP and $\operatorname{MSP}(\mathrm{G}) .{ }^{*} \mathrm{P}<0.05, * * \mathrm{P}<0.01$ compared with the control. 
A



F
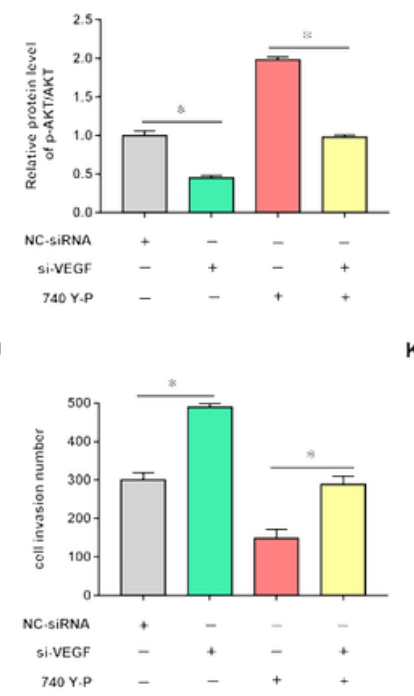

G
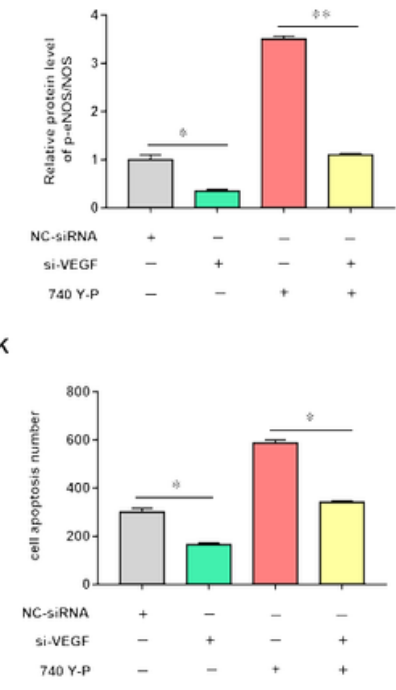



H
D

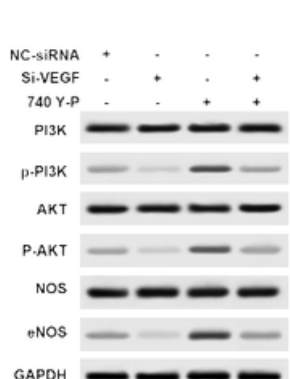



I



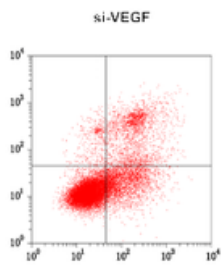
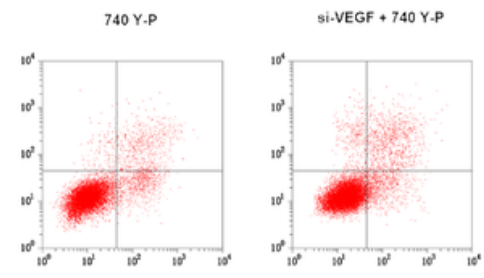

Figure 7

Silencing VEGF inhibits the activation of the PI3K/Akt/eNOS pathway and induced apoptosis of glioma cells. U87 cells were transfected with si-VEGF $(10 \mathrm{nmol} / \mathrm{L})$ and treated with $740 \mathrm{Y}-\mathrm{P}(100 \mu \mathrm{g} / \mathrm{mL})$ for $24 \mathrm{~h}$. Cells were collected for the expression analysis and the biological functional analysis. The mRNA $(A)$ and protein (B and C) levels of VEGF in U87 cells were detected by RT-qPCR and Western blot after treatment with si-VEGF and/or 740 Y-P. Phosphorylation levels of related protein PI3K (E), Akt (F), eNOS (G) of the $\mathrm{PI} 3 \mathrm{~K} / \mathrm{AKK} / \mathrm{eNOS}$ pathway, and eNOS $(\mathrm{H})$ were detected after treatment U87cells with si-VEGF and/or 740 Y-P. After the treatment of U87 cells with si-VEGF and/or 740 Y-P, the proliferation of U87 cells was determined by CCK-8 (I); the invasive cell was detected by Transwell assay (J); cell apoptosis was evaluated by flow cytometry $(K) .{ }^{*}<0.05, * * P<0.01$ compared with the control. 